\title{
Diabetes Reflect Study: Redefining Fundamental Learning of SMBG through Educational Color Tools
}

\author{
Katsunori Suzuki1 ${ }^{*}$, Takaaki Sato1, Mariko Hatta ${ }^{2}$ \\ ${ }^{1}$ Division of Endocrinology and Metabolism, Saiseikai Niigata Daini Hospital, Niigata, Japan \\ ${ }^{2}$ Department of Nutrition, Saiseikai Niigata Daini Hospital, Niigata, Japan \\ Email: ${ }^{*}$ katsu-s@ngt.saiseikai.or.jp
}

Received 3 March 2015; accepted 5 April 2015; published 10 April 2015

Copyright (C) 2015 by authors and Scientific Research Publishing Inc.

This work is licensed under the Creative Commons Attribution-NonCommercial International License (CC

BY-NC).

http://creativecommons.org/licenses/by-nc/4.0/

(c) () (9) Open Access

\begin{abstract}
Aims: To investigate the effects of a newly developed color-coded educational glucose control instruction (COLOR), combined with a color-displayed glucose meter on changes in type-2 diabetes patient's behavior and their HbA1c levels. Material and Methods: Patients using a COLOR-selfmonitoring of blood glucose (SMBG) method were instructed to record daily blood glucose levels with red (high glucose $\geq 180 \mathrm{mg} / \mathrm{dL}$ ) or blue (low glucose $\leq 70 \mathrm{mg} / \mathrm{dL}$ ) colored circles on their SMBG notebooks according to the colors displayed on the glucose meter. Results: COLOR-SMBG showed an improved the actual behavior modification scores by $14 \%$ compared to Conventional group at 1-month follow-up ( $<<0.05)$. The mean HbA1c levels of COLOR-SMBG significantly decreased at 2 months $(0.14 \% \pm 0.28 \%, p<0.01), 4$ months $(0.31 \% \pm 0.48 \%, p=0.05)$, and 6 months $(0.39 \% \pm 0.49 \%, p=0.01)$ follow-up. Conclusions: The results suggest for the first time the usefulness of the instructional intervention using COLOR-SMBG for patients with type 2 diabetes. This trial was registered with UMIN (No. UMIN000011767).
\end{abstract}

\section{Keywords}

Color-Coded Educational Glucose Control Instruction, Education, SMBG, Type 2 Diabetes

\section{Introduction}

The effectiveness of conducting simplified self-monitoring blood glucose (SMBG) for both type-1 and type-2 diabetes patients has already been demonstrated by DCCT [1], UKPDS [2], and the Kumamoto Study [3]. Wel-

${ }^{*}$ Corresponding author.

How to cite this paper: Suzuki, K., Sato, T. and Hatta, M. (2015) Diabetes Reflect Study: Redefining Fundamental Learning of SMBG through Educational Color Tools. International Journal of Clinical Medicine, 6, 227-234.

http://dx.doi.org/10.4236/ijcm.2015.64028 
schen et al. [4], Sarol et al. [5], Norris et al. [6], and St. John et al. [7] reported in their meta-analysis of patients with type 2 diabetes that a more remarkable decrease in HbA1c was observed in the SMBG, while Karter et al. [8] and Ziegler et al. [9] reported that there was an association between the frequency of SMBG and the improvement of HbA1c. SMBG is also recommended in American Diabetes Association (ADA) 2013 Guidelines [10], and the individualization is emphasized so that the frequency of measurement can be set based on the patients' life rhythm. The International Diabetes Federation (IDF) [11] recommends SMBG for patients under noninsulin treatment as well. As such, although the effectiveness of SMBG for all the diabetes treatment is now being clarified regardless of type and stage, the fundamental idea is that it is most important to utilize SMBG not only for measuring blood glucose level but also for making patients refer to the measurement value to look back on their life style and modify their behavior.

SMBG monitors currently on the market have a variety of features that enable several functions such as shortened measurement time, reduced size, minimized blood amount needed for measurement, and the error content display in Japanese [12] [13], all of which seem to provide patients with more improved usability than before. Above all, one of the great features of the model with color (not black-and-white) liquid crystal display is that users can recognize the blood glucose level in a visual way, with the high level of blood glucose being displayed in red and the low level in blue as an index of blood glucose level on the liquid crystal display [14]. In addition, it is useful to visually-handicapped patients with diabetes [15]. Hashimoto et al. [12] reported that patients who used the model had a clear awareness of the color display.

As such, although it has previously been suggested that models with color liquid crystal displays are useful for the change in consciousness of patients under diabetes treatment, there the actual behavior modification process remains difficult, which is commonly seen in clinical practice. Therefore, in this study I investigated whether the combination of the newly developed color educational materials, "COLOR method", and SMBG monitor equipped with "color-indicator function" that displays the levels of blood glucose by different colors can improve behavior modifications, and reduce HbA1c levels of type 2 diabetes patients.

\section{Materials and Methods}

\subsection{Patients and Study Group}

In February 2011, a total of 112 patients with type 2 diabetes who used a color-displayed OneTouch Ultra$\mathrm{Vue}^{\mathrm{TM}}$ (Johnson and Johnson, Life Scan) glucose meter were registered in this study, and randomly assigned to 2 experimental groups: 1) COLOR-SMBG group (56 patients; age: $60.7 \pm 14.8$; SMBG usage period: $4.8 \pm 4.2$ years); and 2) Conventional SMBG group (56 patients; age: $61.0 \pm 14.6$; SMBG usage period: $4.8 \pm 3.4$ years). No significant difference was found between the two groups at the baseline demographic and characteristics (Figure 1). This study was approved by the Saiseikai Niigata Daini Hospital Ethics Committee (receipt number: E10-15; accepted: November 8, 2010). Written informed consent was obtained from all patients before randomization.

\subsection{SMBG with Color-Coded Educational Glucose Control Instruction (COLOR-SMBG)}

The color monitor of the OneTouch UltraVue ${ }^{\mathrm{TM}}$ glucose meter displays the index of the blood glucose level by five stages of colors under the numerical value depending on the degree of the blood glucose levels (blue, pale blue, green, orange, and red). Red or blue color, for example, signifies the blood glucose level $\geq 180 \mathrm{mg} / \mathrm{dL}$ or $\leq$ $70 \mathrm{mg} / \mathrm{dL}$, respectively (Figure 2). Following experimental group assignment, patients of COLOR-SMBG group had a brief instruction of "COLOR method" by medical staff (but not from the author who was blinded to patient group assignment) at the first visit to the outpatient clinic (Figure 3). According to the indicated color next to the glucose reading on the OneTouch UltraVue ${ }^{\mathrm{TM}}$ display, patients were asked to record the blood glucose level and circle the glucose levels with the same color as the glucose meter display onto their self-management notebook published by Japan Association for Diabetes Education and Care (http://www.nittokyo.or.jp) without any additional cost to the patients. An instruction illustrated celluloid sheet, was provided to each patient. Patients in the COLOR-SMBG group were instructed to record daily blood glucose level with a red (blood glucose levels $\geq 180 \mathrm{mg} / \mathrm{dL}$ ) or blue $(\leq 70 \mathrm{mg} / \mathrm{dL}$ ) circle onto the self-management SMBG notebook using the provided color ball-point pen with notebook when the glucose meter indicated these colors at the time of the blood glucose reading (Figure 3). Patients in the Conventional SMBG group were merely asked to record their 


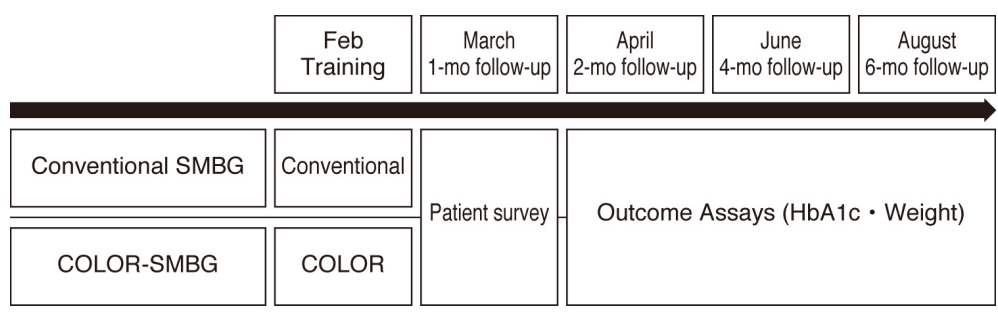

\begin{tabular}{|c|c|c|c|c|c|}
\hline $\begin{array}{c}\text { Experimental } \\
\text { Group }\end{array}$ & $\mathrm{N}$ & SMBG history(Y) & Weight(kg) & $\mathrm{HbA1c}(\%)$ & $\operatorname{Age}(\mathrm{Y})$ \\
\hline $\begin{array}{c}\text { Conventional } \\
\text { SMBG }\end{array}$ & 56 & $\begin{array}{c}4.85 \\
\text { (Range 1-18) }\end{array}$ & $\begin{array}{c}63.47 \\
\text { (Range 40-127) }\end{array}$ & $\begin{array}{c}7.7 \\
\text { (Range 5.4-13.2) }\end{array}$ & $\begin{array}{c}61.02 \\
\text { (Range 23 - 81) }\end{array}$ \\
\hline COLOR-SMBG & 56 & $\begin{array}{c}4.83 \\
\text { (Range 0.5 - 22) }\end{array}$ & $\begin{array}{c}65.78 \\
\text { (Range 42.3-111.2) }\end{array}$ & $\begin{array}{c}7.72 \\
\text { (Range 5.7 - 11.5) }\end{array}$ & $\begin{array}{c}60.73 \\
\text { (Range 27-86) }\end{array}$ \\
\hline
\end{tabular}

Figure 1. Design of reflect study. Data were expressed as median and a range. mo: Months. $\mathrm{N}=112$ (Male 63, Female 49). At the final follow up, it was $\mathrm{N}$ $=43$ (Conventional) and $\mathrm{N}=41$ (Color), with contact lost with 13 Conventional patients and 15 Color patients.

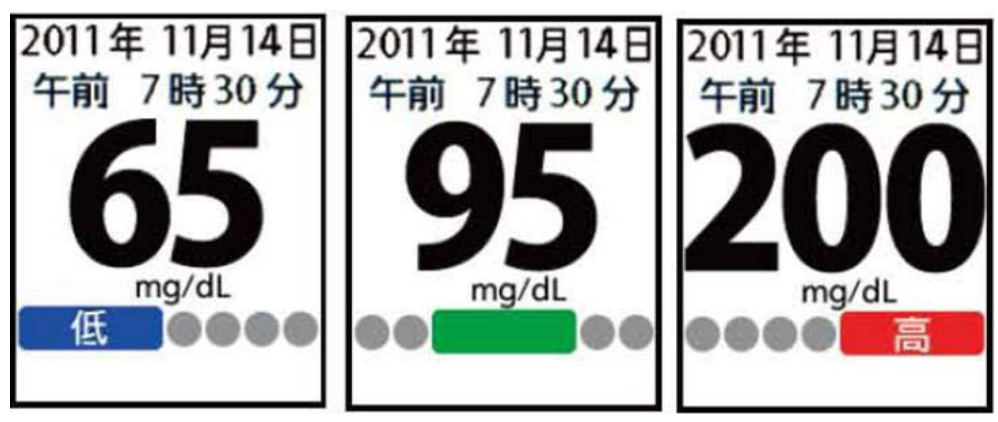

Figure 2. Color range indicator of glucose meter. The first row of the indicator shows year/month/day in Japanese. Second row displays time (hour/minute) in Japanese. Left panel color with kanji character "Low" indicates low blood glucose level with blue-color indicator; middle panel shows normal blood glucose level with green-color indicator; and right panel shows high blood glucose level with red-color with kanji character "High".

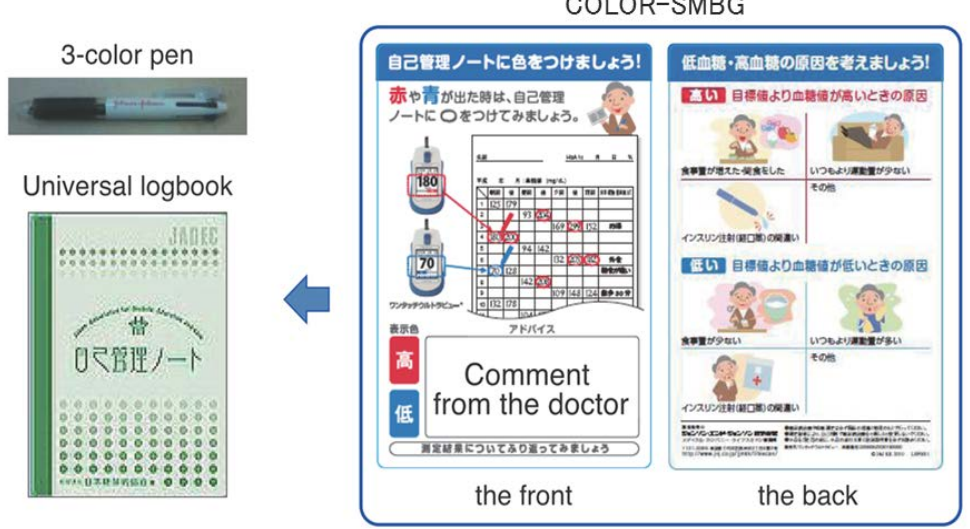

Figure 3. COLOR-SMBG instruction. Patients were provided with a 3-color (red, blue, and black colors) ball point pen (left upper panel), blood glucose logbook (left lower panel, SMBG notebook), and color instructed celluloid sheet, "COLOR instruction", which provides detailed instructions with illustrations so that the patient can follow the instructions without medical staff assistance (right panel). 
daily glucose levels without colored circles. It is noted that the COLOR-SMBG instruction can be completed within several minutes by medical staff at the outpatient clinic. Thus, patients were not required to attend any additional education classes for the SMBG instruction.

One month later (second visit to outpatient clinic), patients were asked to submit the notebook and answer questionnaires (Table 1) for analysis of changes in consciousness and behavior modifications. Patients in the Conventional SMBG group were also asked to submit the notebook and answer the same questionnaires at their second visit to the outpatient clinic.

\subsection{Outcome Measurements}

The obtained questionnaires from patients in both groups were analyzed for the patients' attitude. In each questionnaire, percentage ratios of "Yes/No" or "Very often/Often/Sometimes/Rarely/Very Rarely" were calculated in each group (Table 1 ).

Changes in HbA1c levels (conventional JDS) at 2 months, 4 months, and 6 months follow-up were also analyzed. It is noted that medication and insulin treatment protocols for diabetes remained the same during the study (Figure 1).

\subsection{Statistical Analysis}

Data were presented as median and data range (HbA1c and body weight in Figure 1) or mean \pm standard deviation (SD) (HbA1c change in Figure 4). Wilcoxon rank-sum test was used to compare differences in the patient characteristics and questionnaire between the COLOR-SMBG group and the Conventional SMBG group, and the paired $t$-test was used to determine the change of HbA1c in both groups at each time point. $\mathrm{p}<0.05$ was used to define statistical significance. The statistical analysis was performed using PASW Statistics 18 software (SPSS Hong-Kong, Quarry Bay, Hong Kong).

\section{Results}

All patients completed the questionnaire at one-month follow-up. However, I could not follow up all patients for 6 months due to logistical reasons of patients, and other reasons of patients, such as moving. Therefore, 41 patients in the COLOR-SMBG group and 43 patients in the Conventional SMBG group were completely followed up for 6 months as the end point of the study.

\subsection{Awareness of Color and the Level of Blood Glucose}

Before the "COLOR-SMBG" method was initiated, 100 patients of total 112 patients (89\%) were aware of the

Table 1. Evaluation of interest in blood glucose result by the Conventional SMBG group and the COLOR-SMBG group. Patient survey results (1-month post-training).

\begin{tabular}{|c|c|c|c|c|c|c|c|}
\hline & & Yes & No & & & & $P$ \\
\hline \multirow{3}{*}{$\begin{array}{l}\text { Do you play attention to the 5-level color range } \\
\text { indicator? }\end{array}$} & Conventional & $66 \%$ & $34 \%$ & - & - & - & - \\
\hline & COLOR & $91 \%$ & $9 \%$ & & & & $<0.05$ \\
\hline & & Very Often & Often & Sometimes & Rarely & Very Rarely & \\
\hline \multirow{2}{*}{ Have you recognized high glucose results? } & Conventional & $29 \%$ & $48 \%$ & $11 \%$ & $6 \%$ & $6 \%$ & \\
\hline & COLOR & $53 \%$ & $28 \%$ & $12 \%$ & $2 \%$ & $5 \%$ & $<0.05$ \\
\hline \multirow{2}{*}{$\begin{array}{l}\text { Have you reflected your dietary patterns, exercise, } \\
\text { and/or other lifestyle based on daily BG results? }\end{array}$} & Conventional & $15 \%$ & $44 \%$ & $20 \%$ & $21 \%$ & $0 \%$ & \\
\hline & COLOR & $24 \%$ & $52 \%$ & $19 \%$ & $5 \%$ & $0 \%$ & $<0.05$ \\
\hline \multirow{2}{*}{$\begin{array}{l}\text { Have you ever changed your dietary patterns, } \\
\text { exercise, and/or other lifestyle based on daily } \\
\text { BG results? }\end{array}$} & Conventional & $11 \%$ & $40 \%$ & $14 \%$ & $26 \%$ & $9 \%$ & \\
\hline & COLOR & $5 \%$ & $60 \%$ & $14 \%$ & $19 \%$ & $2 \%$ & $<0.05$ \\
\hline \multirow{2}{*}{$\begin{array}{c}\text { Have your levels of interest to SMBG ever been } \\
\text { influenced by your BG measurement? }\end{array}$} & Conventional & $15 \%$ & $38 \%$ & $44 \%$ & $3 \%$ & $0 \%$ & \\
\hline & COLOR & $16 \%$ & $54 \%$ & $30 \%$ & $0 \%$ & $0 \%$ & $<0.05$ \\
\hline
\end{tabular}




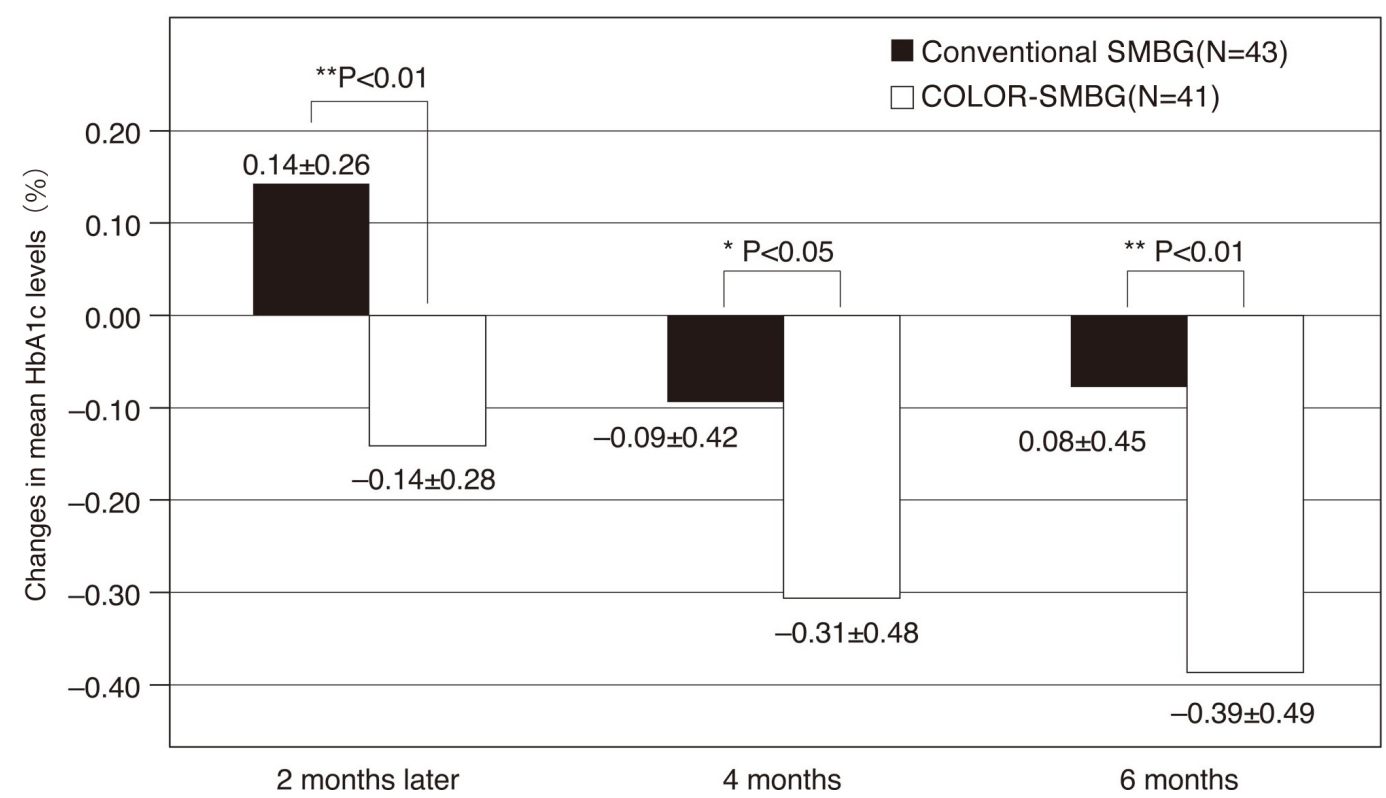

Figure 4. Changes in mean HbA1c levels in Conventional SMBG group and COLOR-SMBG group. The HbA1c levels in Conventional SMBG group and COLOR-SMBG group. ${ }^{* *} \mathrm{p}<0.05$ vs. Conventional SMBG group.

5-level color range indicator OneTouch UltraVue ${ }^{\mathrm{TM}}$ glucose meter. After the COLOR-SMBG intervention, 91\% of patients in the COLOR-SMBG group answered "Yes" to the question "Did you pay attention to the 5-level color range indicator?" while 66\% of patients answered, "Yes" in the Conventional SMBG group $(\mathrm{p}<0.05)$. While $81 \%$ of patients in the COLOR-SMBG group answered "Very Often", or "Often", to the question "Have you recognized high glucose results?" 77\% of patients in the Conventional SMBG group answered "Very often", or “Often” ( $\mathrm{p}<0.05)$ (Table 1).

\subsection{Behavior Modification}

To the question "Have you ever reflected your dietary patterns, exercise and/or other lifestyle by daily BG results?" $76 \%$ of patients in the COLOR-SMBG group answered "Very Often" or "Often" compared to 59\% of patients in the Conventional SMBG group ( $<$ 0.05). In the Conventional SMBG group, 51\% of patients answered "Very Often" or "Often" to the question, "Have you ever changed your dietary patterns, exercise and/or other lifestyle factors based on daily BG results?" whereas 65\% of patients in the COLOR-SMBG group answered "Very Often", or "Often", which was $14 \%$ higher than the Conventional SMBG group $(\mathrm{p}<0.05)$. In the Conventional SMBG group 53\% of patients answered "Very Often", or "Often" to the question, "Has your level of interest in SMBG ever been influenced by your BG measurement?" whereas 70\% of patients in the COLORSMBG group answered "Very Often" or "Often," which was significantly higher than the Conventional SMBG group by $17 \%(\mathrm{p}<0.05)($ Table 1$)$.

\subsection{Change in HbA1c Levels}

In the Conventional SMBG group, mean HbA1c levels were increased $0.14 \pm 0.26$ (SD)\%, at 2 months and then decreased to $-0.09 \% \pm 0.42 \%$, and $-0.08 \% \pm 0.45 \%$, at 4 and 6 months follow-up, respectively. In contrast, mean $\mathrm{HbA1c}$ levels of the COLOR-SMBG group were significantly decreased from pre-intervention to $-0.14 \%$ $\pm 0.28 \%$ ( $p<0.01$, vs. before intervention) at 2 months, and further decreased to $-0.31 \% \pm 0.48 \%(\mathrm{p}<0.05)$ and $-0.39 \% \pm 0.49 \%(\mathrm{p}<0.01)$ at 4 and 6 months, respectively, indicating a significant improvement of blood glucose control in the COLOR-SMBG group (Figure 3).

At 6 months follow-up (endpoint of the study), HbA1c levels were obtained from 43 patients of the Conventional SMBG group and 41 patients of the COLOR-SMBG group.

Thirty-five patients $(76.1 \%)$ of the COLOR-SMBG group significantly decreased HbA1c $(\mathrm{p}<0.05)$, whereas 22 patients (46.9\%) of the conventional SMBG group significantly decreased HbA1c levels. There was a signifi- 
cant difference in numbers of patients whose HbA1c levels were reduced at 6 months follow-up ( $<<0.05)$, indicating the COLOR-SMBG method can improve blood glucose control (Figure 5).

\section{Discussion}

The primary goal of the current study was to make the diabetes patients aware of their daily blood glucose levels, which could improve their actual behavior changes in regards to SMBG resulting in better diabetes control. In this intervention study, we utilized the color display function of the OneTouch UltraVue glucose meter and the COLOR instruction method to improve SMBG quality.

The COLOR-SMBG method significantly reduced HbA1c levels of the diabetes patients. Colors are referred to as visual language. Red, among them all, is especially considered to give a strong impression and likely to be remembered [14]. Along with this human visual recognition effect, the function of OneTouch UltraVue ${ }^{\mathrm{TM}}$ to display the index in red for the blood glucose value $\geq 180 \mathrm{mg} / \mathrm{dL}$ may have helped to make patients more aware of high blood glucose level as a whole. Following the COLOR-SMBG method, 91\% (43 patients) of patients were aware of the function compared to $66 \%$ (32 patients) of patients in the Conventional SMBG group, suggesting that patients in the COLOR-SMBG group maintained a high level of awareness to the display that showed the high/low level of blood glucose in color (Table 1).

The ratio of the patients who modified their behavior in their daily life was 65\% in the COLOR-SMBG group and $51 \%$ in the Conventional SMBG group ( $<<0.05$, Table 1) suggesting that the behavior modification being evoked by the COLOR-SMBG method was significant. A stronger sense of risk for hyper- or hypoglycemia was
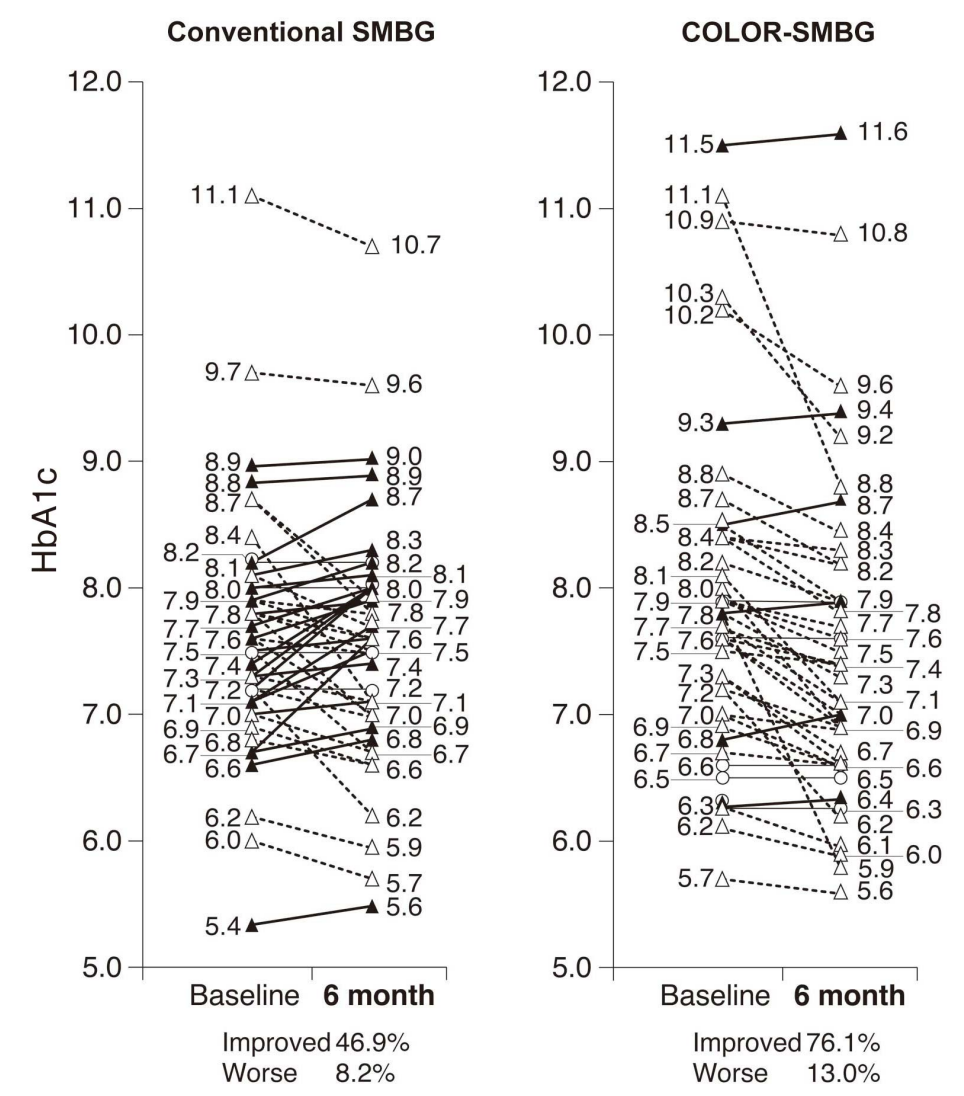

Figure 5. Individual variations of changes in HbA1c levels in Conventional SMBG group and COLOR-SMBG group. Changes in HbA1c levels in each patient between baseline and 6 months follow-up (Decrease in HbA1c: Conventional SMBGN = 22, COLOR-SMBGN = 35, Increase in HbA1c: Conventional SMBGN = 21, COLOR-SMBGN = 6). 
generated by the COLOR-SMBG method among those patients who had a higher degree of cognition and understanding of the blood glucose levels indicated by color codes. Furthermore, the increase in interest to SMBG by COLOR instruction may also improve communication between patient and physician (medical staff) by using simplified color-codes of blood glucose levels, such as "Red decreased" or "No blue color anymore".

With regard to the increase in HbA1c seen at 2 months follow-up in the Conventional SMBG group, the possible involvement of this study being initiated in wintertime might be suggested as one reason. In this post New Year's holiday season in Japanese snowy cold regions, when dietary habit is prone to change, HbA1c is likely to worsen due to the influence of the decrease in activity caused by cold weather. Usually, these cases are often recognized at clinical sites as a seasonal variation observed in wintry days [16]-[18]. It is noted that even at a time of concern of exacerbation of HbA1c level, a decrease in HbA1c levels was observed in the COLOR-SMBG group. There were six patients (17\%) in the COLOR-SMBG group who did not put a circle, but none of them had any increase in HbA1c. This can be attributable to the fact that the COLOR-SMBG method significantly enhanced the awareness of these patients to SMBG.

\section{Efficacy of Instructional Intervention}

Studies have already been conducted aiming for visual effect due to color, some of which have reported that patient's awareness for the state of high/low blood glucose levels was raised by utilizing OneTouch UltraVueglucose meter with the function to display the index of the measured blood glucose level in color [12] [13]. However, there is no precedent of the intervention studies using additional tools in order to make patients voluntarily aware of colors. Therefore, the current study is the first study aiming to prove the efficacy of the color-codes of blood glucose levels.

In the current study, I conducted an instructive intervention, "COLOR-SMBG" study to raise more awareness to color and gathered objective data, which showed remarkable effects. Although it is widely recognized that SMBG is effective to the patients with diabetes, regardless of it being a treatment with insulin or non-insulin, the actual efficacy is said to lead the patients to behavior modification [19] [20]. As is shown by the result of the current study, it seems that the changes in patient's behavior modification was as a consequence of instructions such as using OneTouch UltraVue ${ }^{\mathrm{TM}}$ with the "COLOR-SMBG method = color notifying function", putting a colored circle on the self-management notebook by patients themselves, and their physician's asking colorthemed questions such as "How many red circles?" as a supportive means during examination at the outpatient clinic.

A great amount of effort and time are generally required to carry out an efficient, reliable patient instruction. Whereas in this intervention study of the COLOR-SMBG method, behavior modification occurred in patients by only utilizing several minutes long instruction with a simple tool without causing any difference in the individual instruction contents, which additionally improved HbA1c as well. In the COLOR-SMBG method, instructions can be offered in a short period of time without being accompanied by the increase of medical expense due to dose increase/addition or the increase in the number of measurements. It is conceivable that this method enables us to improve the blood glucose level effectively without causing economic burden on patients even in the limited time period of SMBG in a conventional examination. This study was closed for ethical reasons 6 months later. It is unexpectedly surprising that the COLOR-SMBG method showed a significant beneficial effect at 2 months and lasted as far as 6 months (endpoint of the study). Further studies are necessary to prove the longterm effects of the COLOR-SMBG method on HbA1c levels and SMBG quality. (No changes are being made to the diabetes medication, so it is thought to be because of the COLOR-SMBG instruction.)

\section{Conclusion}

Results suggest for the first time the usefulness of the instructional intervention using a color-coded blood glucometer and the "COLOR-SMBG" method for patients with type 2 diabetes. This simple, patient-friendly, and highly effective method should be able to enhance the quality of SMBG further. Longer-term effects of the combined color-coded blood glucose meter and the COLOR-SMBG method on SMBG should be investigated.

\section{Acknowledgements}

A part of this study was presented in abstract form at the 72nd American Diabetes Association scientific session, 
United States, June, 2011. There were no potential conflicts of interest relevant to the article.

\section{References}

[1] Diabetes Control and Complications Trial (DCCT) Research Group (1993) The Effect of Intensive Treatment of Diabetes on the Development and Progression of Long-Term Complications in Insulin-Dependent Diabetes Mellitus. New England Journal of Medicine, 329, 977-986. http://dx.doi.org/10.1056/NEJM199309303291401

[2] United Kingdom Prospective Diabetes Study (UKPDS) Group (1998) Intensive Blood-Glucose Control with Sulfonylureas or Insulin Compared with Conventional Treatment and Risk of Complications in Patients with Type 2 Diabetes (UKPDS 33). Lancet, 352, 837-853. http://dx.doi.org/10.1016/S0140-6736(98)07019-6

[3] Ohkubo, Y., Kishikawa, H., Araki, E., Miyata, T., Isami, S., Motoyoshi, S., et al. (1995) Intensive Insulin Therapy Prevents the Progression of Diabetic Micro Vascular Complications in Japanese Patients with Non-Insulin-Dependent Diabetes Mellitus: A Randomized Prospective 6-Year Study. Diabetes Research and Clinical Practice, 28, 103-117. http://dx.doi.org/10.1016/0168-8227(95)01064-K

[4] Welschen, L.M., Bloemendal, E., Nijpels, G., Dekker, J.M., Heine, R.J., Stalman, W.A., et al. (2005) Self-Monitoring of Blood Glucose in Patients with Type 2 Diabetes Who Are Not Using Insulin: A Systematic Review. Diabetes Care, 28, 1510-1517. http://dx.doi.org/10.2337/diacare.28.6.1510

[5] Sarol Jr., J.N., Nicodemus Jr., N.A., Tan, K.M. and Grava, M.B. (2005) Self-Monitoring of Blood Glucose as Part of a Multi-Component Therapy among Non-Insulin Requiring Type 2 Diabetes Patients: A Meta-Analysis (1966-2004). Current Medical Research and Opinion, 21, 173-184. http://dx.doi.org/10.1185/030079904X20286

[6] Norris, S.L., Engelgau, M.M. and Narayan, K.M. (2001) Effectiveness of Self-Management Training in Type 2 Diabetes: A Systematic Review of Randomized Controlled Trials. Diabetes Care, 24, 561-587. http://dx.doi.org/10.2337/diacare.24.3.561

[7] St. John, A., Davis, W.A., Price, C.P. and Davis, T.M. (2010) The Value of Self-Monitoring of Blood Glucose: A Review of Recent Evidence. Journal of Diabetes and Its Complications, 24, 129-141. http://dx.doi.org/10.1016/j.jdiacomp.2009.01.002

[8] Karter, A.J., Ackerson, L.M., Darbinian, J.A., D’Agostino Jr., R.B., Ferrara, A., Liu, J., et al. (2001) Self-Monitoring of Blood Glucose Levels and Glycemic Control: The Northern California Kaiser Permanente Diabetes Registry. The American Journal of Medicine, 111, 1-9. http://dx.doi.org/10.1016/S0002-9343(01)00742-2

[9] Ziegler, R., Heidtmann, B., Hilgard, D., Hofer, S., Rosenbauer, J. and Holl, R., DPV-Wiss-Initiative (2011) Frequency of SMBG Correlates with HbA1c and Acute Complications in Children and Adolescents with Type 1 Diabetes. Pediatric Diabetes, 12, 11-17. http://dx.doi.org/10.1111/j.1399-5448.2010.00650.x

[10] American Diabetes Association (2013) Standards of Medical Care in Diabetes-2013. Diabetes Care, 36, S11-S66. http://dx.doi.org/10.2337/dc13-S011

[11] International Diabetes Federation (2005) Global Guideline for Type 2 Diabetes. http://www.idf.org/guidelines/type-2-diabetes

[12] Hashimoto, N., Hirota, Y., Sakaguchi, K., et al. (2010) Examination of Usefulness of Color Indicator Function of OneTouch UltraVue ${ }^{\mathrm{TM}}$. Medicine and Pharmacy, 63, 637-642.

[13] Urata, K., Yonaga, K., Hirakawa, M., et al. (2010) Usage Evaluation of Self-Monitoring Blood Glucose Monitor OneTouch UltraVue ${ }^{\mathrm{TM}}$ in Patients with Type 2 Diabetes. Practice, 27, 691-695.

[14] Minami, R. (2003) “How to Utilize Color” for Nursing Care. Gendai Shorin, Tokyo, 109-132.

[15] Ijima, H., Nakamura, M., Miyano, M., et al. (2011) Examination of Visibility and Instruction Method for the Visually Impaired Who Has Difficulty with the Operation of SMBG Measurement. Diabetes, 54, S-213.

[16] Doi, K. (2010) Relationship between the New Year Holidays and HbA1c. Obesity and Diabetes, 9, $254-255$.

[17] Lshii, H., Suzuki, H., Baba, T., Nakamura, K. and Watanabe, T. (2001) Seasonal Variation of Glycemic Control in Type 2 Diabetic Patients. Diabetes Care, 24, 1503. http://dx.doi.org/10.2337/diacare.24.8.1503

[18] Sakura, H., Tanaka, Y. and Iwamoto, Y. (2010) Seasonal Fluctuations of Glycated Hemoglobin Levels in Japanese Diabetic Patients. Diabetes Research and Clinical Practice, 88, 65-70. http://dx.doi.org/10.1016/j.diabres.2009.12.011

[19] Norris, S.L., Lau, J., Smith, S.J., Schmid, C.H. and Engelgau, M.M. (2002) Self-Management Education for Adults with Type 2 Diabetes: A Meta-Analysis of the Effect on Glycemic Control. Diabetes Care, 25, 1159-1171. http://dx.doi.org/10.2337/diacare.25.7.1159

[20] Training Guidebook for Diabetologist. 4th Edition, Japan Diabetes Society, Shindan \& Chiryou, Tokyo, 70. 